\title{
Are relatives of patients with multiple HNPCC spectrum tumours at increased risk of cancer?
}

\author{
S R Brown, P J Finan, D T Bishop
}

\begin{abstract}
Background-Relatives of patients with early onset colorectal cancer, a feature of hereditary non-polyposis colorectal cancer (HNPCC), are at increased risk of colorectal cancer.

Aims-To investigate risk in relatives of patients with multiple primary cancers, another feature of HNPCC.

Methods-Details were obtained on patients from one region who had developed colorectal cancer and a separate primary from the HNPCC tumour spectrum (colorectal, stomach, urinary, ovary, endometrial). Overall, 157 patients had second primaries occurring between 1990 and 1995 and 128 completed family histories were obtained by structured interview (study group). A comparison group of 444 patients with a single colorectal cancer were similarly interviewed.

Results-Fifteen families (13\%) from the study group were suggestive of HNPCC compared with three $(0.7 \%, p<0.0001)$ from the comparison group. Overall risk of colorectal cancer in close relatives of the study group was 3.4 times the general population rate compared with 1.8 times for the comparison group. Bowel cancer risk was even higher for relatives of bowel/ ovary and bowel/endometrial subgroups, but was similar to the comparison group for the bowel/bowel subgroup. Finally, extracolonic HNPCC associated cancers were seen twice as frequently as expected in the general population in relatives of the study group.

Conclusion-This study highlights the importance of taking a family history in patients with multiple primary cancers and indicates the risk of malignancy in their relatives.

(Gut 1998;43:664-668)
\end{abstract}

Keywords: hereditary non-polyposis colorectal cancer; family history; relative risk

Up to one in five patients with colorectal cancer report a positive family history. ${ }^{1-3} \mathrm{~A}$ small proportion of these patients have recognised hereditary syndromes such as familial adenomatous polyposis (FAP) and hereditary non-polyposis colorectal cancer (HNPCC). However, by far the majority of familial cancer patients show no clear mode of inheritance. Familial clustering in some of these patients is presumably due to a combination of chance and shared environmental factors. There remains the possibility that a substantial pro- portion could be the result of genetic susceptibility: similarity to either HNPCC or other common but weakly penetrant genes.

In HNPCC, patients inherit a germline mutation in one of the mismatch repair genes. $^{4-6}$ Clinically, this predisposes to early onset (usually less than 50 years of age) colon cancer which is predominantly right sided. In addition, affected individuals are prone to develop multiple primary cancers, from both the colorectum and a broad spectrum of extracolonic sites. These sites include stomach, small bowel, urinary tract, ovary, and endometrium. ${ }^{7}$

Recognition of clinical features found in HNPCC (other than family history) may allow identification of patients with an increased genetic susceptibility to colorectal cancer. Early onset colorectal cancer is one such feature and studies have shown close relatives of such people to be at substantially increased risk of colorectal cancer as well as being very likely to belong to an HNPCC family. ${ }^{8}$

Predisposition to multiple primary cancers is another feature of both HNPCC and many other hereditary cancer syndromes. In the general colorectal cancer population, if multiple primary cancers are primarily the result of hereditary factors, there would be an increased cancer risk in relatives. This increase should be greater than that associated with each site alone.

To test this hypothesis of an increased cancer risk, we investigated the family histories of cancer in patients from one region who have developed multiple primaries from the HNPCC tumour spectrum. The cancer risk in relatives was compared with the risk for relatives of a population of consecutive single colorectal cancer patients attending one consultant surgeon's practice.

Patients and methods

A group of probands from one health region who had developed both colorectal cancer and at least one other cancer from the HNPCC spectrum between 1990 and 1995 were identified by the Yorkshire Cancer Organisation. The tumour spectrum for the second primary included colorectal, stomach, small bowel, urinary, ovarian, and endometrial cancers. After obtaining ethical approval, consent was sought from all consultant general surgeons within Yorkshire for release of information from the Yorkshire Cancer Registry concerning their relevant patients. Only patients with histologically proved colorectal cancer not arising in the context of FAP or inflammatory bowel disease, and a confirmed separate primary, not 
Table 1 Criteria used to assess the contribution of dominantly inherited susceptibility to cancer

Amsterdam criteri

Histologically verified colorectal cancer in three relatives, one a first degree relative of the other two

One colorectal cancer diagnosed before the age of 50 years

Two or more generations affected

Familial adenomatous polyposis excluded

Less strict criteria for HNPCC

Colorectal cancer in two first degree relatives

One colorectal cancer diagnosed before the age of 50 years

Consistent with dominant inheritance

Familial adenomatous polyposis excluded

Plus at least one of:

A third colorectal cancer

Endometrial cancer, small bowel cancer, ureteric cancer

Early onset (aged less than 50 years) ovarian, stomach, bladder, or hepatobiliary carcinoma

A third relative under 45 years of age with an adenoma or significant polyp $(>1 \mathrm{~cm}$,

tubulovillous or villous, or with severe dysplasia) at any age

From Hall et al. ${ }^{8}$

representative of local or metastatic spread, were included. Because there were substantially more patients with multiple colorectal cancers, this group was limited to those registered as having developed a second colorectal cancer between 1992 and 1995.

For each proband, the relevant consultant and general practitioner were informed about the proposed contact with their patient. A standard letter explaining the nature of the study and requesting a personal interview to draw up a family tree, was sent to each patient or surviving next of kin or spouse.

A home interview was carried out by a trained interviewer. Information collected included dates of birth and death, causes of death, and diagnoses of cancer in all first degree (parents, siblings, and children), second degree (grandparents, uncles, and aunts) and, where possible, more distant relatives. Considerable effort was made to confirm all suspected diagnoses of cancer by consulting cancer registries, histology reports, death certificates, and hospital notes and by obtaining the assistance of general practitioners and hospital consultants. Because of the difficulty of confirmation of cancer diagnoses from the early part of the century in parents of probands, both confirmed and probable diagnoses (where there was reliable information from family members but no medical verification) were included in the analysis. When the situation arose that the proband had died, the nearest next of kin was approached. However, this was only after obtaining relevant permission from both the consultant and the next of kin's general practitioner.

Table 2 Demographic data for the populations of multiple and single primary cancers

\begin{tabular}{llll}
\hline & $\begin{array}{l}\text { No of } \\
\text { patients }\end{array}$ & $\begin{array}{l}\text { Median (range) age (y) at } \\
\text { diagnosis of first colorectal } \\
\text { cancer }\end{array}$ & $\begin{array}{l}\text { Colorectal tumour } \\
\text { site (\% right sided) }\end{array}$ \\
\hline Group 1 (comparison group) & 444 & $69.5(27-91)$ & 26 \\
Group 2 (study group) & 128 & $69.0(33-93)$ & $44 \dagger$ \\
Group 2cr & 49 & $68.5(35-90)$ & $62^{\star} \dagger$ \\
Group 2st & 40 & $73.0(50-93)$ & 34 \\
Group 2ov & 31 & $64.5(33-92)$ & 35 \\
Group 2en & 28 & $68.0(44-91)$ & 30 \\
Group 2ur & 5 & $68.0(42-82)$ & 40 \\
Group 2sb & 3 & $71.0(69-83)$ & 50 \\
\hline
\end{tabular}

«This percentage includes $10(19 \%)$ patients with synchronous cancers of both the left and right sides of the large bowel.

†Significant increase compared with the single primary group.
As a comparison group, consecutive colorectal cancer patients from one consultant's colorectal practice attending between 1987 and 1997 were similarly interviewed. Patients with documented evidence of a second primary cancer from the HNPCC tumour spectrum, as well as those with colitis and FAP associated colorectal cancer were excluded. This gave a cohort of single HNPCC primary cancer patients.

For each of the two groups, the observed risk of colorectal cancer in first degree relatives was compared with the expected incidence of colorectal cancer. The expected incidence was calculated by summing the individual risk for each relative (based on Yorkshire Cancer Registry data on age and sex specific incidence rates $\left.^{9}\right)$. In addition, an assessment of the contribution of dominantly inherited susceptibility to colorectal and other cancers (suggestive of HNPCC) was made using both the Amsterdam criteria and less stringent criteria modified from Hall et al (see table 1). ${ }^{8}$ These less stringent criteria were formulated to address some of the limitations of the Amsterdam criteria. These criteria add weight to the occurrence of extracolonic cancers and certain colorectal adenomas within a family member.

Mean age at occurrence of the first colorectal cancer was compared for each of the two groups using Student's $t$ test. Colorectal tumour site and frequency of familial aggregation were compared using the $\chi^{2}$ test.

\section{Results}

POPULATION DATA

A total of 592 patients underwent colorectal cancer surgery under one consultant over the 10 year period analysed and have been approached for a family history. Four were excluded from analysis because of inflammatory bowel disease, one because of a family history of FAP, and four because they were adopted. A further 56 patients had died and had no traceable next of kin while 34 patients and 15 next of kin refused to give a history. This left 478 patients ( $82 \%$ of the cohort) where a detailed family history was obtained and verified. Thirty four of these patients had documented evidence of a multiple primary cancer from the HNPCC tumour spectrum. These patients were also excluded to give a comparison group of 444 single primary cancer patients.

A total of 166 patients registered with the Yorkshire Cancer Registry met our multiple primary cancer criteria. Consent to approach $157(96 \%)$ of these patients was obtained from their relevant consultants. Complete family histories were obtained in 128 of these 157 patients $(82 \%)$, forming the study group (group 2). Twenty seven patients had no family history obtained: nine were untraceable and two patients, 11 next of kin, four general practitioners, and one consultant refused contact. In two patients, only limited information concerning family history was available. Demographic data including age of onset of the first colorectal cancer and tumour location for 
Table 3 Familial aggregation for the population of the comparison (group 1) and study groups (group 2)

\begin{tabular}{|c|c|c|c|c|c|c|}
\hline \multirow[b]{2}{*}{ Group } & \multirow{2}{*}{$\begin{array}{l}\text { No of } \\
\text { patients }\end{array}$} & \multicolumn{4}{|c|}{ First degree relatives with colorectal cancer } & \multirow{2}{*}{$\begin{array}{l}\text { HNPCC } \\
\text { (meeting criteria }\end{array}$} \\
\hline & & 0 & 1 & 2 & 3 & \\
\hline Group 1 & 444 & $347(78 \%)$ & $74(17 \%)$ & $16(4 \%)$ & 0 & $3(0.7 \%)$ \\
\hline Group 2 & 128 & $94(75 \%)$ & $24(16 \%)$ & $5(4 \%)$ & $5(4 \%)$ & $16(13 \%) \dagger$ \\
\hline Group 2cr & 42 & $29(69 \%)$ & $11(26 \%)$ & 0 & $2(5 \%)$ & $4(10 \%) \ddagger$ \\
\hline Group 2st & 28 & $21(75 \%)$ & $6(21 \%)$ & 0 & $1(4 \%)$ & $1(4 \%)$ \\
\hline Group 2ov & 26 & $21(80 \%)$ & $2(8 \%)$ & $1(4 \%)$ & $2(8 \%)$ & $5(20 \%)^{\star}$ \\
\hline Group 2en & 24 & $15(63 \%)$ & $5(21 \%)$ & $4(17 \%)$ & 0 & $4(17 \%)$ \\
\hline Group 2ur & 5 & $4(80 \%)$ & 0 & 0 & $1(20 \%)$ & $1(20 \%)$ \\
\hline Group 2sb & 3 & $2(67 \%)$ & $1(33 \%)$ & 0 & 0 & 0 \\
\hline
\end{tabular}

Significantly different to the comparison group: ${ }^{\star} \chi^{2}=29.51, \mathrm{df}=1, \mathrm{p}<0.00001 ;+\chi^{2}=36.21, \mathrm{df}=1, \mathrm{p}<0.00001 ; \neq \chi^{2}=17.62, \mathrm{df}=1$, $\mathrm{p}<0.00001 ; \delta \chi^{2}=33.68, \mathrm{df}=1, \mathrm{p}<0.00001$.

this study group were no different to the comparison single primary cancer group with available family histories.

For the study group (group 2) there were 42 probands with two or more colorectal cancers (group 2cr), 28 with colorectal and stomach cancer (group 2st), 26 with colorectal and ovarian cancer (group 2ov), 24 with colorectal and endometrial cancer (group 2en), five with colorectal and urinary cancer (group 2 ur), and three with colorectal and small bowel cancer (group 2sb).

DEMOGRAPHIC DATA

There was no difference in the age distribution at time of diagnosis of the first colorectal cancer between the study and comparison groups. In addition, the age distribution was not significantly different between each of the multiple primary subgroups. However, there was a significant difference in the tumour site distribution. Forty four per cent of the study group had a first colorectal cancer proximal to the splenic flexure, compared with only $26 \%$ of the single primary comparison group $\left(\chi^{2}\right.$ $=22.30, \mathrm{df}=1, \mathrm{p}<0.0001)$. This difference only occurred in the subgroup of patients with multiple colorectal cancers where $62 \%$ of the initial colorectal cancers were right sided $\left(\chi^{2}=26.52\right.$, $\mathrm{df}=1, \mathrm{p}<0.0001)$. Ten of these multiple colorectal cancer patients $(19 \%)$ had synchronous lesions in both the right and left sides of the large bowel at initial diagnosis. However, even excluding these cases there remained a signifi-

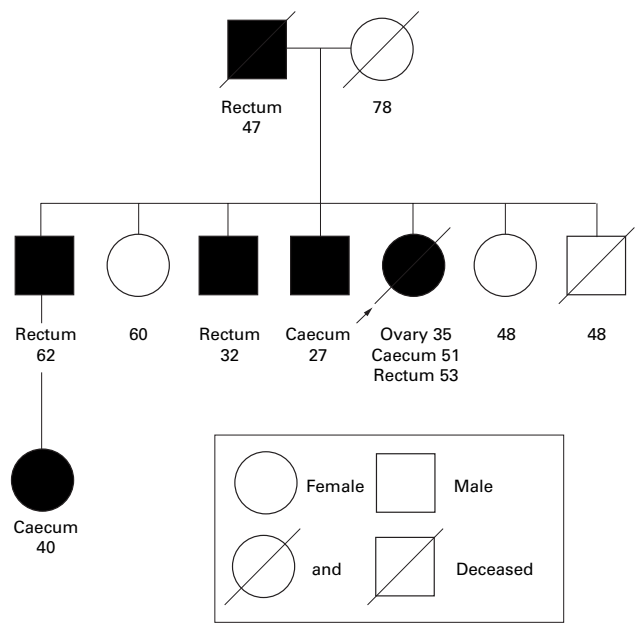

Figure 1 Pedigree from the multiple primary group that is consistent with a diagnosis of HNPCC. cantly higher proportion of initial right sided lesions (20/39 patients, $\chi^{2}=18.07, \mathrm{df}=1$, $\mathrm{p}<0.0001)$. Tumour site distribution was similar to the comparison group for the other multiple primary subgroups (table 2 ).

FAMILIAL AGGREGATION

For the multiple primary study group, 24 probands $(16 \%)$ had one first degree relative with colorectal cancer (21 confirmed), five (4\%) had two affected first degree relatives (four confirmed), and five (4\%) had three or more affected first degree relatives (all diagnoses confirmed), a total of 50 first degree relatives (46 diagnoses confirmed). Table 3 details familial aggregation for each of the subgroups. The frequency of familial cancer was similar to that of the comparison group, although there were more patients in the study group with three or more affected first degree relatives.

INCIDENCE OF SUSPECTED HNPCC

For the study group, 10 (8\%) families fulfilled the Amsterdam criteria for diagnosis of HNPCC and an additional six (5\%) were con-

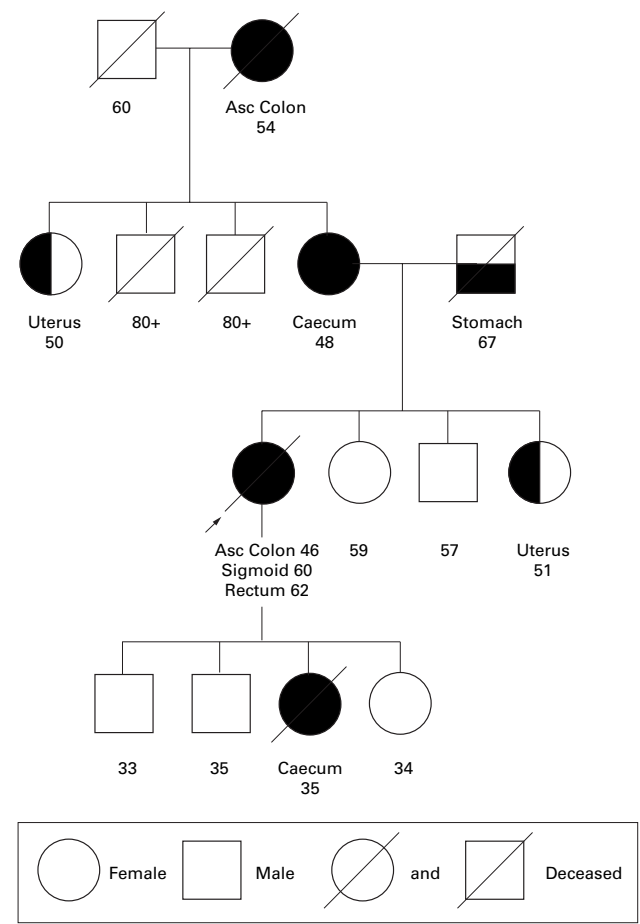

Figure 2 Pedigree from the multiple primary group that is consistent with a diagnosis of HNPCC. 


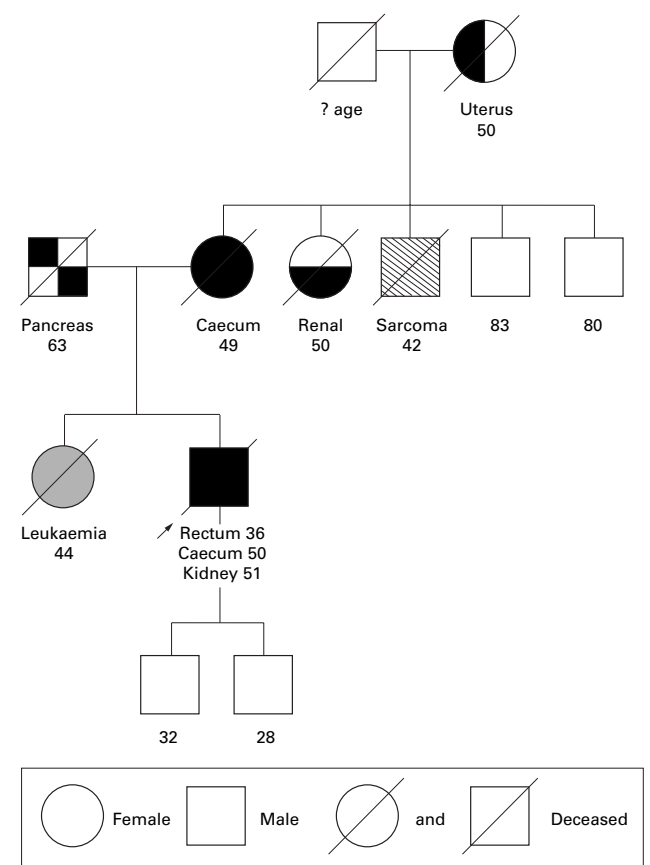

Figure 3 Pedigree from the multiple primary group that is consistent with a diagnosis of HNPCC.

sidered likely to be HNPCC on the basis of the less strict criteria. Therefore, a total of 16 $(13 \%)$ of the families where pedigree information was available, were likely to be HNPCC. This contrasts with only three families $(0.7 \%)$ from the comparison group, which were considered likely to be HNPCC. Figures 1-3 illustrate three pedigrees identified in the study. Table 3 details the incidence of these putative HNPCC families in each of the groups.

RELATIVE RISK OF COLORECTAL CANCER

There was a total of 722 first degree relatives of the study group probands. Fifty $(7 \%)$ had colorectal cancer (46 confirmed). In an age and sex matched regional population the expected number of colorectal cancer cases was 15.7. Therefore, the relative risk in the first degree relatives of the study group was $3.2(\mathrm{p}<0.0001$ for testing true relative risk different from 1.0) using confirmed cancer data. Including probable cases, the relative risk was $3.4(\mathrm{p}<0.0001)$. Using confirmed data from the single primary comparison group, the relative risk for colorectal cancer was $1.6(\mathrm{p}<0.0001)$ and, including probable cases was $1.8(\mathrm{p}<0.0001)$. Some of
Table 5 Risk of extracolonic cancers in first degree relatives of the comparison (group 1) and study (group 2) groups compared with the general population

\begin{tabular}{|c|c|c|c|c|c|c|}
\hline & \multicolumn{6}{|c|}{ Extracolonic tumours } \\
\hline & \multicolumn{3}{|l|}{ HNPCC } & \multicolumn{3}{|c|}{ Non-HNPCC } \\
\hline & Observed & Expected & $R R$ & Observed & Expected & $R R$ \\
\hline Group 1 & 67 & 78.1 & 0.9 & 218 & 252.5 & 0.9 \\
\hline Group 2 & 32 & 14.8 & $2.1^{\star}$ & 77 & 67.2 & 1.1 \\
\hline
\end{tabular}

Cancers associated with HNPCC include those of stomach, small bowel, endometrium, ovary, and urinary tract.

${ }^{\star}$ Significant result using Poisson distribution $(\mathrm{p}<0.001)$.

the more recently treated comparison group patients had only been followed up for a short time. Because some of these patients may go on to develop a second primary cancer, relative risk analysis was carried out on those seen during the first five year period (1987-1992) who have been followed up for at least 60 months and have not developed a second HNPCC cancer. The relative risk for colorectal cancer for these patients was exactly the same as for the comparison group (table 4).

Table 4 also shows the relative risk of colorectal cancer for each of the subgroups. The urinary and small bowel subgroups were excluded because of insufficient numbers for meaningful analysis. Colorectal cancer risk for first degree relatives was increased for all other groups. However, risk was substantially increased in groups 2 ov and 2en, where there was a sixfold and fivefold increase in risk, respectively, over the general population. The risk for relatives in groups $2 \mathrm{cr}$ and $2 \mathrm{st}$ was similar to that seen in relatives of the comparison group.

RELATIVE RISK OF EXTRACOLONIC CANCERS

The relative risk of extracolonic HNPCC and non-HNPCC cancers was examined for the comparison and study groups. In the comparison single primary group, both HNPCC associated and non-HNPCC associated extracolonic cancers were seen at the expected frequency (table 5). In the multiple primary study group extracolonic non-HNPCC cancers were also seen at the expected frequency. However, HNPCC associated extracolonic cancers were seen at over twice the frequency expected in the general population.

\section{Discussion}

This study confirms several previous studies showing that relatives of patients with colorectal cancer are at increased risk of developing

Table 4 Risk of colorectal cancer in first degree relatives compared with the general population

\begin{tabular}{|c|c|c|c|c|c|}
\hline \multirow[b]{2}{*}{ Group } & \multirow[b]{2}{*}{ No of patients } & \multicolumn{3}{|c|}{ Colorectal cancer in first degree relatives } & \multirow[b]{2}{*}{$p$ Value ${ }^{*}$} \\
\hline & & Observed & $\begin{array}{l}\text { Expected in general } \\
\text { population }\end{array}$ & Relative risk & \\
\hline Group 1 (comparison group) & 444 & 41 & 23.1 & 1.8 & $<0.0005$ \\
\hline Group 1 (first 5 years) & 190 & 21 & 11.7 & 1.8 & $<0.001$ \\
\hline Group 2 (study group) & 128 & 50 & 15.7 & 3.2 & $<0.00001$ \\
\hline Group 2cr & 42 & 12 & 5.8 & 2.1 & 0.02 \\
\hline Group 2st & 28 & 9 & 3.9 & 2.3 & 0.02 \\
\hline Group 2ov & 26 & 15 & 2.5 & 6.1 & $>0.00001$ \\
\hline Group 2en & 24 & 13 & 2.5 & 5.1 & $>0.00001$ \\
\hline
\end{tabular}

$\star$ Poisson distribution.

Relative risk was calculated by comparing the incidence of colorectal cancer in relatives with the expected age and sex matched incidence in the general population. 
colorectal cancer themselves. ${ }^{3} 1011$ Combining all previous estimates of risk for first degree relatives of colorectal cancer patients gives a consistent figure of about two times the population risk. ${ }^{12}$ However, we have shown that relatives of patients with multiple primary cancers seem to be at even higher risk. The overall relative risk was around twice that of our cohort of single colorectal cancer patients. We also observed that this risk was different for each of the subgroups of primary cancers. Colorectal cancer risk in relatives of the multiple colorectal subgroup as well as the colorectal/stomach subgroup approximated that of the single colorectal cancer group. However, risk was over five times that of the general population risk for relatives of the ovarian and endometrial subgroups.

A major determinant of this observed increased risk may be the selection of patients with a hereditary predisposition to colorectal cancer. The finding of a significantly higher incidence of suspected HNPCC families in the multiple primary group compared with the single primary group, seems to confirm this.

A particular feature of colorectal tumours from HNPCC patients is their tendency to be right sided. This is in contrast to the left sided predominance in the general colorectal cancer population. ${ }^{13}$ Analysis of tumour site showed that over $60 \%$ of the patients subsequently developing a second colorectal primary, had a right sided tumour initially. This has been noted in other studies ${ }^{14}$ and again suggests a similar genetic susceptibility to HNPCC in these families.

One further observation was made in our multiple primary cancer families, indicating a similar hereditary susceptibility to HNPCC. The incidence of HNPCC associated extracolonic cancers was noted to be increased in relatives of multiple primary cancer patients. This same increased risk was not observed in non-HNPCC associated cancers, nor was it seen in the single primary cancer group.

Identification of genetic susceptibility in patients with cancer has major practical implications with regard to screening, treatment, and follow up. Relatives of patients with HNPCC have a $50 \%$ chance of inheriting the gene mutation. Those with the germline mutation have an $80 \%$ chance of developing colorectal cancer. They also have a substantially increased risk of developing other cancers. ${ }^{15}{ }^{16}$ Screening of these patients should be intensive, with some recommending at least two yearly colonoscopies. ${ }^{17}$ Many also recommend screening for extracolonic cancers in the form of regular abdominal, pelvic, and vaginal sonography for families with a tendency to develop these cancers. ${ }^{13} 18$

Patients who develop multiple primary cancers should initially have a careful, thorough family history taken. This will identify those from HNPCC families and allow appropriate screening, as well as referral for genetic counselling and possible mutation analysis. However, even in those where HNPCC has been excluded, there is still a substantial risk of colorectal cancer in first degree relatives, warranting screening colonoscopy. For relatives of patients with colorectal and endometrial or ovarian cancer, the lifetime risk of developing colorectal cancer indicated by our study is greater than 1 in $10^{19}$ and colonoscopic surveillance is therefore particularly important.

The authors would like to thank all the clinicians who allowed their patients to be interviewed, as well as the Yorkshire Cancer Registry who provided information on the patients with multiple primary cancers. Special thanks go to Sue Haynes, Patricia ple primary cancers. Special thanks go to Sue Haynes, Patricia Mack, and Bette Ward who interviewed the majority of patients and their families. This work was supported by the Imperial Cancer Research Fund and was presented at the Association of
Surgeons, Bournemouth, UK, in April 1997.

1 Lovett E. Family studies in cancer of the colon and rectum. Br 7 Surg 1976;63:13-18.

2 Ponz de Leon M, Sassatelli R, Sacchetti C, et al. Familial aggregation of tumours in the three-year experience of a population-based colorectal cancer registry. Cancer Res 1989;49:4344-8.

3 Stephenson BM, Finan PJ, Gascoyne J, et al. Frequency of familial colorectal cancer. Br F Surg 1991;78:1162-6.

4 Leach FS, Nicolaides NC, Papadopoulos N, et al. Mutations of a mutS homologue in hereditary colon cancer. Cell 1993; 75:1215-25.

5 Liu B, Parsons RE, Hamilton SR, et al. HMSH2 mutations in hereditary nonpolyposis colorectal cancer kindreds. in hereditary nonpolyposis

6 Mary J-L, Bishop T, Kolodner R, et al. Mutation analysis of the $\mathrm{hMSH} 2$ gene reveals a three base pair deletion in a family predisposed to colorectal cancer development. Hum Mol Genet 1995;3:2067-9.

7 Watson P, Lynch HT. Extracolonic cancer in hereditary nonpolyposis colorectal cancer. Cancer 1993;71:677-85.

8 Hall NR, Finan PJ, Ward B, et al. Genetic susceptibility to colorectal cancer in patients under 45 years of age. $B r f$ Surg 1994;81:1485-9.

9 Joslin C, Rider L, Round C, eds. Yorkshire Cancer Registry: Report for the year 1991, including cancer statistics for 1984-1988. Leeds: Yorkshire Regional Cancer Organisation, 1992

10 St John DJB, McDermott FT, Hopper JL, et al. Cancer risk in relatives of patients with common colorectal cancer. Ann Intern Med 1993;118:785-90.

11 Fuchs CS, Giovannucci EL, Colditz GA, et al. A prospective study of family history and the risk of colorectal cancer. $N$ Engl f Med 1994;331:1669-74.

12 Bishop DT, Thomas HJ. The genetics of colorectal cancer. Cancer Surv 1990;9:585-604.

13 Lynch HT, Smyrk T. Hereditary nonpolyposis colorectal cancer (Lynch syndrome). An updated review. Cancer 1996;78:1149-67.

14 Svendsen LB, Bülow S, Mellemgaard A. Metachronous colorectal cancer in young patients: expression of the hereditary nonpolyposis colorectal cancer syndrome? Dis Colon Rectum 1991;34:790-3.

15 Bailey-Wilson JE, Elston RC, Schuelke GS, et al. Segregation analysis of hereditary nonpolyposis colorectal cancer. Genet Epidemiol 1986;3:27-38.

16 Scapoli C, Ponz de Leon M, Sassatelli R, et al. Genetic epidemiology of hereditary nonpolyposis colorectal cancer syndromes in Modena, Italy: results of a complex segregation analysis. Ann Hum Genet 1994;58:275-95.

17 Vasen HFA, Mecklin J-P, Khan PM. Interval cancers in hereditary nonpolyposis colorectal cancer. Lancet 1995; 345:1183-4.

18 Vasen HFA, Wijnen JT, Menko FH, et al. Cancer risk in families with hereditary nonpolyposis colorectal cancer diagnosed by mutation analysis. Gastroenterology 1996;110: 1020-7.

19 Houlston RS, Murday V, Haracopos C, et al. Screening and genetic counselling for relatives of patients with colorectal cancer in a family cancer clinic. BMF 1990;301:366-8. 